Relato de Caso

\title{
Efeito de um protocolo de intervenção baseado na aprendizagem motora para marcha e equilíbrio em jovem com esclerose lateral primária: um estudo de caso
}

\author{
Effect of an intervention protocol based on motor learning for walking and balance \\ in young patient with primary lateral sclerosis: a case study
}

\author{
João Antonio da Silva Filho ${ }^{1}$, Clara Inés García García ${ }^{2}$, \\ Julialba Castellanos Ruiz ${ }^{3}$, Mónica Yamile Pinzón Bernal ${ }^{3}$
}

\begin{abstract}
Silva Filho JA, García García CI, Castellanos Ruiz J, Pinzón Bernal MY. Efeito de um protocolo de intervenção baseado na aprendizagem motora para marcha e equilíbrio em jovem com esclerose lateral primária: um estudo de caso / Effect of an intervention protocol based on motor learning for walking and balance in young patient with primary lateral sclerosis: a case study. Rev Med (São Paulo). 2021 jan.-fev.;100(1):70-7.
\end{abstract}

RESUMO: Introdução: A esclerose lateral primária (ELP) é uma doença rara caracterizada por uma degeneração do neurônio motor superior ocorrendo geralmente na sexta década de vida com um início insidioso de uma paresia espástica simétrica, lentamente progressiva, frequentemente começando em membros superiores e inferiores. A prática de exercícios pode trazer benefícios a estes sujeitos levando a uma aprendizagem motora (AM) e melhorando o repertório motor insuficiente. Objetivo: Determinar o efeito de um protocolo de intervenção baseado na AM para melhorar a marcha e equilíbrio em jovem com ELP. Metodologia: É um estudo de caso composto por uma mulher, 29 anos, solteira, colombiana, branca, submetida à avaliação pré-teste (1 sessão), intervenção de protocolo (18 sessões) e pós teste (1 sessão). Para coleta de dados foram utilizados a Escala de Tinetti, Teste de Romberg, Timed Get Up And Go, Teste de alcance funcional, Baropodometria e Estabilometria. Resultados: A Escala de Tinetti (reação postural) mostrou melhora para balaço em um pé; na Escala de Tinetti (marcha) os resultados positivos foram encontrados em largura, simetria, continuidade e direção do passo; Testes de Romberg mostraram evolução para estabilidade sobre superfície plana com olhos fechados e sobre espuma de Fomy com olhos abertos; no teste Timed Get Up \& Go o tempo de percurso foi reduzido de 13,64 segundos para 12,5 segundos. Conclusão: Após a aplicação do protocolo de intervenção baseado na AM houve avanços funcionais em marcha e equilíbrio de jovem com ELP.

Palavras-chave: Aprendizagem; Marcha; Equilíbrio postural; Esclerose lateral.

\begin{abstract}
Introduction: Primary lateral sclerosis (PLS) is a rare disease characterized by the upper motor neuron degeneration that usually begin in the sixth decade of life. It has an insidious onset of symmetrical, slowly progressive spastic paresis, which often initially affects upper and lower limbs. The practice of exercises may benefit people with PLS by motor learning (ML) and improving their poor motor repertoire. Objective: To determine the effect of a ML-based intervention protocol to improve walking and balance in a young woman with PLS. Methodology: This is a case report of PLS in a 29-year-old Caucasian female, single, Colombian, submitted to pre-test evaluation ( 1 session), ML-based protocol intervention (18 sessions) and post-test evaluation (1 session). For data collection, the Tinetti Scale, Romberg test, Timed Get Up And Go, Functional Range test, Baropodometry and Stabilometry were used. Results: The Tinetti Scale (postural reaction) showed improvement in a foot swing; in the Tinetti Scale (walking) the positive results were found in width, symmetry, continuity and direction of the walk; Romberg Tests showed evolution for stability on flat surfaces with closed eyes and on Fomy foam with open eyes; in the Timed Get Up \& Go Test the travel time was reduced from 13.64 seconds to 12.5 seconds. Conclusion: After the application of the protocol of intervention based on ML there were functional advances in gait and balance of young with ELP.
\end{abstract}

Keywords: Learning; Gait; Postural balance; Lateral sclerosis.

Estudo realizado durante estágio de docência e pesquisa de João Antonio S. Filho na Universidad Autónoma de Manizales (UAM), enquanto mestrando em Ciências da Reabilitação pela Universidade Federal do Rio Grande do Norte (UFRN).

1. Programa de Pós-Graduação em Ciências da Reabilitação - Universidade Federal do Rio Grande do Norte (UFRN) - Santa Cruz (RN), Brasil. ORCID: https://orcid.org/0000-0002-5486-5080. E-mail: joaofilho.pb@hotmail.com.

2. Fisioterapeuta da Fundación IPS UAM, Manizales (Caldas), Colômbia. ORCID: https://orcid.org/0000-0003-4866-1054. E-mail: clarai.garciag@ autonoma.edu.co.

3. Fisioterapeutas. Professoras e pesquisadoras da Universidad Autónoma de Manizales (UAM). Manizales (Caldas), Colômbia. ORCID: Ruiz JC https://orcid.org/0000-0002-4857-7330; Bernal MYP - https://orcid.org/0000-0003-4678-2346.E-mails: jcastellanos@autonoma.edu.co; myamile@ autonoma.edu.co.

Endereço para correspondência: João Antonio S. Filho. Rua João Batista de Amorim, 387. Bairro Novo - Guarabira, PB, Brasil. CEP: 58.200-000. E-mail: joaofilho.pb@hotmail.com. 


\section{INTRODUÇÃO} esclerose lateral primária (ELP) é uma doença
rara caracterizada por degeneração do neurônio motor superior ocorrendo geralmente na sexta década de vida com início insidioso de uma paresia espástica simétrica, lentamente progressiva, frequentemente começando em membros superiores e inferiores. Comparada com a esclerose lateral amiotrófica (ELA), a ELP tem uma taxa de progressão mais lenta e um prognóstico de sobrevida de mais de 10 anos a partir do início dos sintomas ${ }^{1-4}$. Estudos estimam que aproximadamente $2 \%$ a $5 \%$ dos pacientes de clínicas neuromusculares adultas são ELP², com maior prevalência masculina ${ }^{6}$ e podem apresentar sintomas como espasticidade, hiperreflexia, hipertonia, rigidez articular, fraqueza muscular, déficit cognitivo, de coordenação e equilíbrio ${ }^{5}$.

À medida que os sintomas avançam, a independência funcional sofre um declínio podendo incapacitar o indivíduo na realização de atividades de vida diária (AVD's) ${ }^{6}$. A prática de exercícios pode trazer benefícios levando a uma aprendizagem motora (AM) e melhorando o repertório motor insuficiente. A AM é descrita como uma mudança relativamente permanente, decorrente da experiência e prática, na capacidade do indivíduo para realizar uma determinada habilidade motora. ${ }^{7}$

Uma avaliação detalhada se faz necessária para identificar as barreiras e facilitadores no processo de reabilitação, entretanto, há escassez na literatura de trabalhos que abordem a atuação fisioterapêutica na ELP, principalmente baseada nos princípios da AM. Sendo assim, surgiu a necessidade de realizar este estudo através da realização de testes no Laboratório de Movimento Humano da Universidad Autónoma de Manizales (UAM), Colômbia, com o objetivo de determinar o efeito de um protocolo de intervenção baseado na AM para melhorar a marcha e equilíbrio em jovem com ELP. A disponibilização de um protocolo com base nas fundamentações da AM voltado a indivíduos com ELP poderá beneficiar não somente os pacientes com este diagnóstico, como também fisioterapeutas que atuam na neurorreabilitação.

\section{MATERIAIS E MÉTODOS}

\section{Sujeito}

A pesquisa é experimental, do tipo estudo de caso, composta por uma mulher, 29 anos, solteira, colombiana, branca, assistente administrativa, pertencente ao regime de seguridade social contributivo (sistema de saúde colombiano). Deu entrada no Serviço Fundação IPS UAM dia 13 de julho de 2015 com diagnóstico de transtorno extrapiramidal e do movimento, apresentando espasticidade em isquiotibiais, fraqueza muscular generalizada, marcha lenta com passos curtos e pouca amplitude de movimento em dorsiflexão e flexão plantar, ocasionando vários tropeços. Realizou fisioterapia durante o ano de 2015 obtendo melhoras neuromotoras, no entanto, precisou encerrar o tratamento devido à limitação do seu serviço de saúde. Em seis de abril de 2017, retornou ao serviço com o novo diagnóstico de ELP, ainda com espasticidade em membros inferiores, encurtamento bilateral de flexores plantares e alteração do padrão normal da marcha. Este estudo foi aprovado pelo Comitê de Bioética ${ }^{\circ}$ 080-2018 da Universidad Autónoma de Manizales (UAM). A paciente assinou o Termo de Consentimento Livre e Esclarecido (TCLE) autorizando sua participação voluntária no estudo.

\section{Instrumentos de medidas}

Escala de Tinetti ${ }^{8}$ para a avaliação da reação postural e estabilidade durante a deambulação. Esta é uma escala que avalia simultaneamente a marcha e o equilíbrio, estes dois aspectos oferecem informações sobre o risco de quedas e determinam se há alterações que requerem intervenção. Está composta por nove itens de equilíbrio e sete de marcha. As respostas podem variar entre 0,1 e 2 para execuções anormais, com compensação e normais, respectivamente. A pontuação máxima do equilíbrio é 16 e a da marcha 12 , sendo indicativo de alto risco de quedas a soma de ambos menor que 19.

Teste de Romberg ${ }^{9}$ modificado com olhos fechados e abertos, em superfície plana e sobre espuma para avaliação da organização sensorial, sendo cada condição pontuada entre 0 , referente a incapaz, e 3 , referente a estável. O teste original é focado na investigação do desequilíbrio e dificuldade para a realização da marcha com o paciente em posição bípede com olhos fechados. O resultado positivo deste teste indica lesão na via proprioceptiva.

Velocidade da marcha avaliada de acordo com o tempo cronometrado que o sujeito utiliza, bem como a quantidade de passos, para caminhar 6 metros em linha reta.

Timed Get Up And Go $o^{10,11}$ avalia o risco de quedas, cujo desempenho está relacionado com marcha, equilíbrio e capacidade funcional. É considerado normal quando o tempo de percurso é inferior a 10 segundos para levantar-se de cadeira sem apoios para antebraços, percorrer 3 metros em linha reta, girar, caminhar de volta ao ponto inicial e sentar-se novamente.

Teste de alcance funcional ${ }^{12}$ considera o paciente em posição ortostática, descalço, com a coluna ereta, olhar fixo no horizonte, com membros superiores em flexão de ombros a $90^{\circ}$ e hemicorpo direito rente à parede. É pedido ao sujeito que se estique o máximo que puder, mantendo os pés fixos ao solo, realizando flexão do tronco e respeitando a mesma posição original dos membros superiores; a partir disso verifica-se a distância percorrida em centímetros atingida pelas mãos do indivíduo.

Baropodometria e Estabilometria ${ }^{13}$ são testes 
complementares e objetivos que registram as pressões plantares e estabilidade estática sobre uma plataforma de força P6000 e duas plataformas de pressão ou baropodométricas P-walk composta de sensores. Também foram utilizadas seis câmeras optoeletrônicas Dx 6000, duas câmeras de vídeo digital vista e um eletromiógrafo sem fio de oito canais FREEEMG100.

\section{Procedimentos}

O protocolo utilizado foi baseado nos princípios da AM para melhorar a marcha e equilíbrio de paciente com ELP (Quadro 1). O protocolo foi aplicado no Serviço Fundação IPS UAM, durante seis semanas (nove de agosto de 2018 a 20 de setembro de 2018), sendo realizadas três sessões semanais em dias intercalados, com duração de 50 minutos cada sessão. A paciente foi avaliada inicialmente ao tratamento no dia 21 de junho de 2018 e ao final, em 28 de setembro de 2018 .

Quadro 1 - Protocolo baseado nos princípios da AM para melhorar marcha e equilibrio de paciente com ELP

\begin{tabular}{|c|c|c|}
\hline SEMANAS & SEMANA 1 & SEMANA 2 \\
\hline ATIVIDADES & $\begin{array}{l}\text { 1. Sente-se e levante-se * } \\
\text { 2. Mantenha a posição do ortostatismo por } 30 \\
\text { segundos com os olhos abertos } * * \\
\text { 3. Mantenha a posição do ortostatismo por } 30 \\
\text { segundos com os olhos fechados } * * * \\
\text { 4. Execute marcha estática } * * * *\end{array}$ & $\begin{array}{l}\text { 1. Caminhe para frente um percurso de } 5 \text { metros } \\
\text { sem obstáculos * } \\
\text { 2. Caminhe lateralmente um percurso de } 5 \text { metros } \\
\text { sem obstáculos ** }\end{array}$ \\
\hline RECOMENDAÇÕES & $\begin{array}{l}\text { * Use uma cadeira sem suporte para o antebraço. } \\
\text { Comece a tarefa sentada, com as mãos apoiadas } \\
\text { nas coxas e pés alinhados e descansando no chão. } \\
\text { Saia da cadeira usando a força de suas pernas e, } \\
\text { se necessário, use também a força de suas mãos } \\
\text { para projetar-se para frente e para cima. Depois de } \\
\text { levantar, espere cerca de } 2 \text { segundos e sente-se na } \\
\text { cadeira. Realize esta tarefa (sentar-se e levantar-se) } \\
\text { por algumas vezes até que ela seja finalizada em um } \\
\text { minuto ou, antes de } 1 \text { minuto, se você atingir seu } \\
\text { limite de fadiga. } \\
\text { ** Em pé (ortostatismo) procure um ponto fixo à } \\
\text { sua frente e tome-o como referência para manter } \\
\text { o queixo em linha horizontal. Tente ficar em uma } \\
\text { posição ereta com boa postura corporal, braços } \\
\text { pendurados e dispostos ao lado do corpo, por } 30 \\
\text { segundos. Se você não puder completar o tempo, } \\
\text { sente-se, descanse e tente novamente. Se for muito } \\
\text { difícil manter o tempo no primeiro dia, faça a tarefa } \\
\text { por } 15 \text { segundos e tente os } 30 \text { segundos novamente. } \\
\text { *** Esta tarefa segue as mesmas orientações da } \\
\text { tarefa anterior com o diferencial de que agora você } \\
\text { deve manter seus olhos fechados. } \\
\text { **** Levante-se e faça movimentos com as } \\
\text { pernas como se fosse caminhar sem sair do lugar, } \\
\text { levantando os joelhos bem alto. Suas mãos devem } \\
\text { estar apoiadas na cintura. Complete a tarefa em } 30 \\
\text { segundos. } \\
\text { Obs.: Seu objetivo é concluir a tarefa, não há } \\
\text { necessidade de realizar sessões ou repetições. }\end{array}$ & $\begin{array}{l}\text { * Escolha um local que tenha espaço suficiente } \\
\text { e apresente uma superfície plana. Peça a alguém } \\
\text { para ajudar a verificar as imagens do espaço usando } \\
\text { uma fita métrica ou esteira e marque o início e o } \\
\text { fim de uma caminhada de } 5 \text { metros em linha reta } \\
\text { no chão (com fita adesiva). É nesse espaço que } \\
\text { você deve caminhar a uma velocidade que você } \\
\text { costuma caminhar, o mais natural possível e sem } \\
\text { apoio. Faça a tarefa da melhor maneira possível e } \\
\text { no ritmo normal. } \\
\text { ** Para esta tarefa toma como base as } \\
\text { recomendações anteriores. A única adaptação é } \\
\text { que você deve fazer o passeio andando de lado. } \\
\text { Use o tempo de } 1 \text { minuto de descanso entre as } \\
\text { duas tarefas. } \\
\text { Obs.: Para ambas as tarefas, é recomendável que } \\
\text { você as execute algumas vezes até que o tempo de } \\
5 \text { minutos seja atingido. Neste momento, faça os } \\
\text { percursos de ida e volta que considere necessárias } \\
\text { para completar o tempo. Não acelere seus passos } \\
\text { para fazer várias caminhadas nestes } 5 \text { minutos, o } \\
\text { objetivo do exercício não é quantas vezes você } \\
\text { conseguirá fazer, mas sim treinar a marcha para } \\
\text { executá-la com a mais alta qualidade que você } \\
\text { conseguir. }\end{array}$ \\
\hline
\end{tabular}


Silva Filho JA, et al. Efeito de um protocolo de intervenção baseado na aprendizagem motora para marcha

Quadro 1 - Protocolo baseado nos princípios da AM para melhorar marcha e equilibrio de paciente com ELP

continuação

\begin{tabular}{|c|c|c|}
\hline SEMANAS & SEMANA 3 & SEMANA 4 \\
\hline \multirow{2}{*}{ ATIVIDADES: } & $\begin{array}{l}\text { 1. Caminhe para frente um percurso de } 5 \text { metros } \\
\text { com obstáculos* }\end{array}$ & $\begin{array}{l}\text { 1. Caminhe para frente um percurso de } 10 \text { metros } \\
\text { com obstáculos* }\end{array}$ \\
\hline & $\begin{array}{l}\text { 2. Caminhe lateralmente um percurso de } 5 \text { metros } \\
\text { com obstáculos** }\end{array}$ & $\begin{array}{l}\text { 2. Caminhe lateralmente um percurso de } 10 \text { metros } \\
\text { com obstáculos** }\end{array}$ \\
\hline RECOMENDAÇÕES & $\begin{array}{l}*, * * \text { Para percorrer um percurso de obstáculos, } \\
\text { você pode usar cones, bengalas ou pequenas caixas } \\
\text { no chão com espaço entre elas que permita que } \\
\text { você dê o passo livre, superando as alturas (levante } \\
\text { a perna bem alto para evitar tropeçar). Use as } \\
\text { recomendações dadas para a semana anterior. O } \\
\text { objetivo é estimular passos cada vez mais altos e } \\
\text { pasadas mais distantes. }\end{array}$ & $\begin{array}{l}\text { *, ** Siga as mesmas recomendações da semana } \\
\text { anterior, mas agora você deve estabelecer um } \\
\text { espaço de percurso mais longo ( } 10 \text { metros). }\end{array}$ \\
\hline SEMANAS & SEMANA 5 & SEMANA 6 \\
\hline \multirow{2}{*}{ ATIVIDADES } & $\begin{array}{l}\text { 1. Caminhe para frente um percurso de } 10 \text { metros, } \\
\text { com obstáculos e com um objeto em suas mãos* }\end{array}$ & $\begin{array}{l}\text { 1. Caminhe para frente um percurso de } 10 \text { metros, } \\
\text { com obstáculos e com um objeto em suas mãos* }\end{array}$ \\
\hline & $\begin{array}{l}\text { 2. Caminhe lateralmente um percurso de } 10 \text { metros, } \\
\text { com obstáculos e com um objeto em suas mãos** }\end{array}$ & $\begin{array}{l}\text { 2. Caminhe lateralmente um percurso de } 10 \text { metros, } \\
\text { com obstáculos e com um objeto em suas mãos** }\end{array}$ \\
\hline RECOMENDAÇÕES & $\begin{array}{l}\text { *** Repetir a mesma informação da semana } \\
\text { anterior, no entanto, agora você deve executar as } \\
\text { tarefas segurando uma bola com ambas as mãos, } \\
\text { colocadas na frente do seu corpo (flexão do ombro } \\
\text { em aproximadamente } 90^{\circ} \text {, extensão total dos } \\
\text { cotovelos, punhos neutros). }\end{array}$ & $\begin{array}{l}*, * * \text { Repita as mesmas recomendações da semana } \\
\text { anterior. }\end{array}$ \\
\hline
\end{tabular}

Fonte: Silva Filho et al., 2018.

\section{RESULTADOS}

De acordo com a Escala de Tinetti (reação postural) a paciente obteve 18 pontos antes do tratamento e 14 após, sobre um total de 24 pontos, o que mostra um padrão alterado de equilíbrio estático e dinâmico mesmo após a intervenção, apresentando melhoras para balanço em um pé e pioras para levantar-se da cadeira, balanço no momento de posicionar-se e manter-se de pé, alcançar um objeto alto e sentar-se em uma cadeira (Gráfico 1).

A Escala Tinetti (marcha) apresentou três e cinco pontos, pré e pós-teste, respectivamente, sobre um total de nove, com resultados positivos em largura, simetria, continuidade e direção do passo e negativos em girar enquanto caminha e início do passo (Gráfico 2).

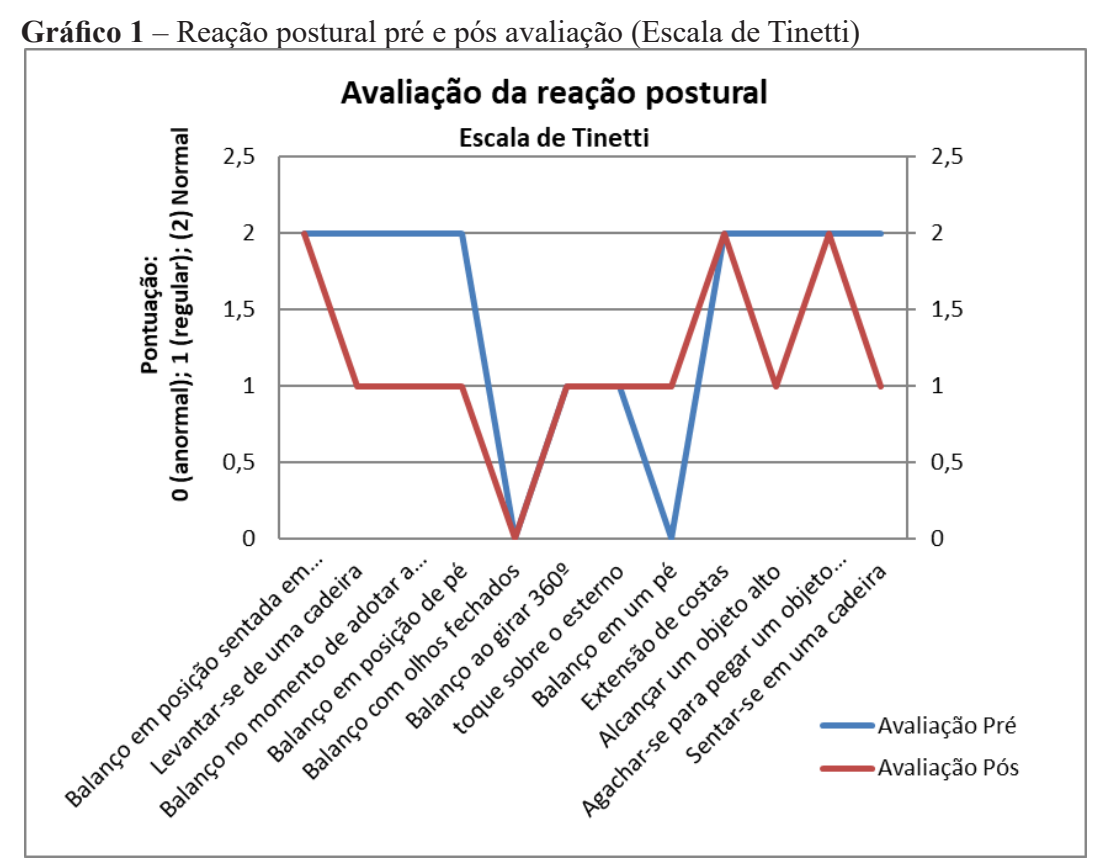

Fonte: Laboratorio Análisis de Movimiento - Universidad Autónoma de Manizales. 
Gráfico 2 - Estabilidade durante a marcha pré e pós avaliação (Escala de Tinetti)

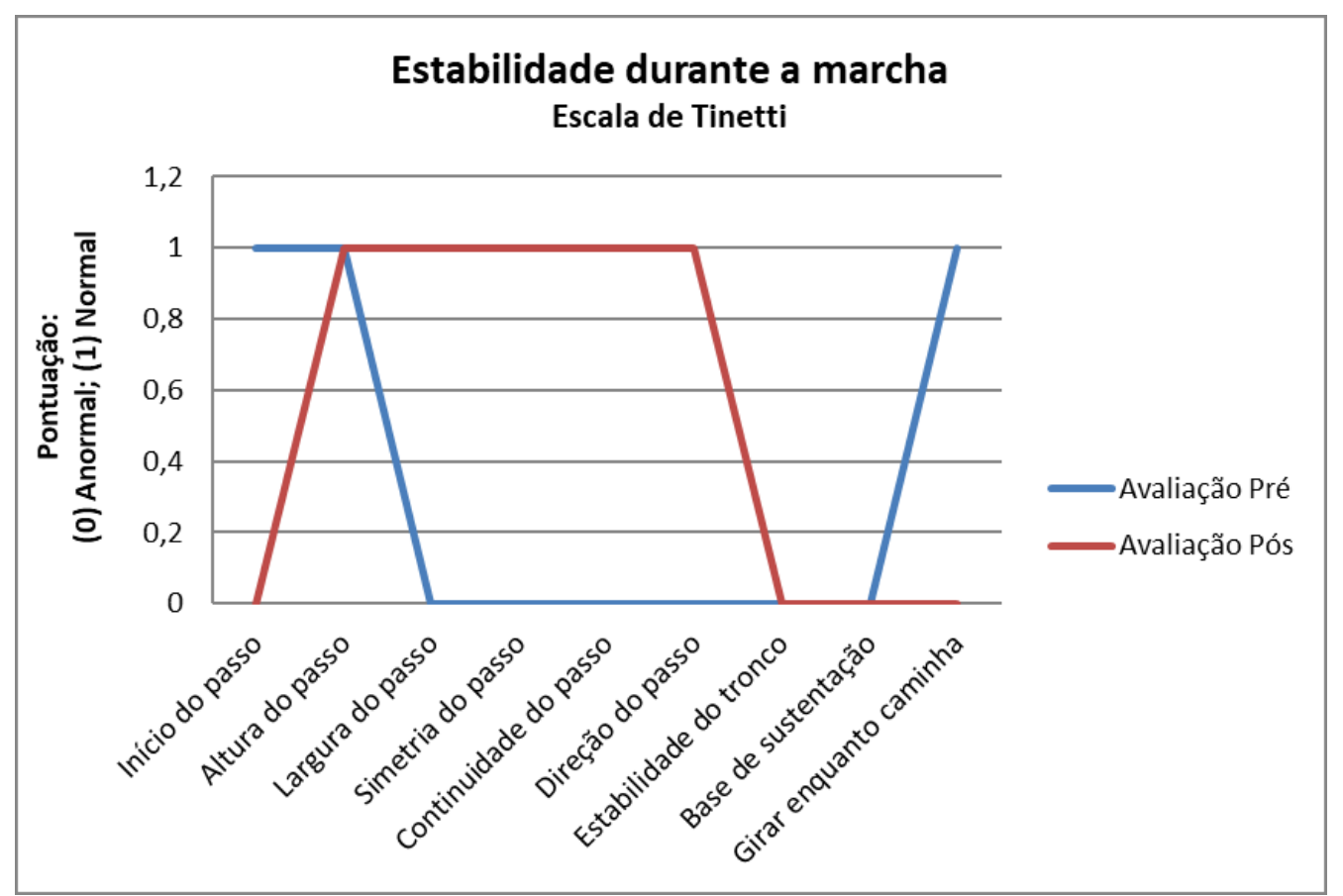

Fonte: Laboratorio Análisis de Movimiento - Universidad Autónoma de Manizales.

A usuária mostrou-se estável nos Testes de Romberg com olhos abertos sobre superfície plana e sobre espuma de Fomy, antes e após a intervenção. Sobre superfície plana com olhos fechados mostrou evolução, saindo de menos de 30 segundos na posição em pé (pré-teste) para mais de
30 segundos: estável (pós-teste). A melhora também ficou evidente sobre espuma de Fomy com olhos abertos após o tratamento, saindo de incapaz de realizar para manter-se estável menos de 30 segundos (Gráfico 3).

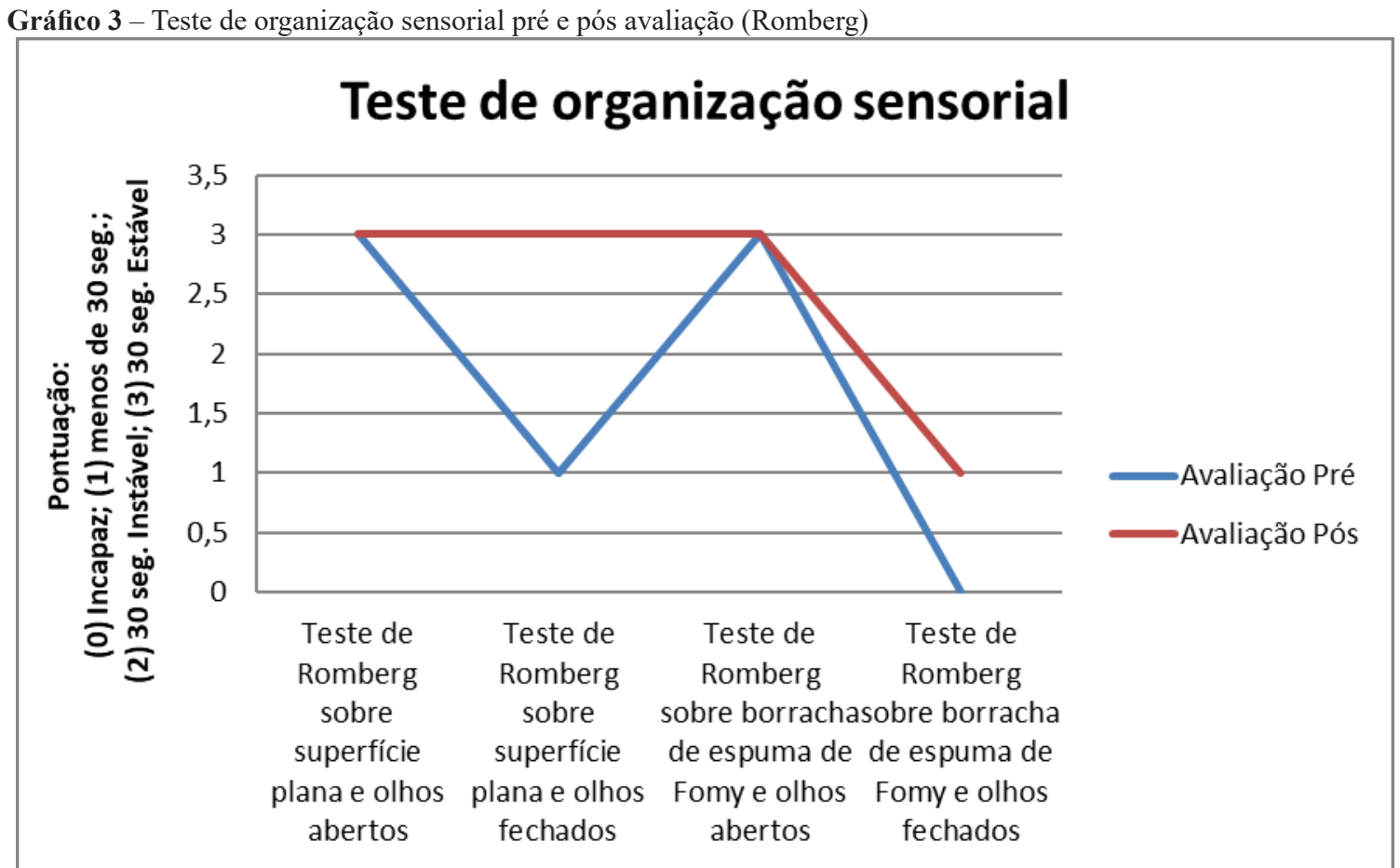

Fonte: Laboratorio Análisis de Movimiento - Universidad Autónoma de Manizales. 
Silva Filho JA, et al. Efeito de um protocolo de intervenção baseado na aprendizagem motora para marcha

Na velocidade da marcha não houve melhoras no número de passos para percorrer seis metros, sendo 16 no pré-teste e 14 no pós-teste, bem como no tempo para percorrer $6 \mathrm{~m}: 6,9 \mathrm{seg}$ e $8,5 \mathrm{seg}$, no pré e pós-teste, respectivamente. No entanto, houve redução do tempo de percurso no teste Timed Get Up \& Go; 13,64 seg e 12,5 seg, pré e pós-teste, respectivamente. O Teste de Alcance Funcional não mostrou melhora, pois houve redução da distância percorrida: $38,85 \mathrm{~cm}$ (pré-teste); 34,25 cm (pósteste).
De acordo com a Baropodometria, no pré-teste, observou-se uma pressão muito forte em pé esquerdo, mais precisamente em região de $2^{\circ}$ e $3^{\circ}$ metatarsos e face medial de calcanhar. No pós-teste, notou-se que o pé esquerdo ainda sofria maior pressão, porém em $4^{\circ}$ e $5^{\circ}$ metatarsos.

$\mathrm{Na}$ Estabilometria, os resultados foram obtidos com a usuária de olhos abertos, pois com olhos fechados não foi possível realizar. Observou-se que as oscilações láteromediais foram mais evidentes que as ântero-posteriores, no pré-teste e no pós-teste, no entanto, neste último as oscilações foram menores (Figuras 1 e 2).

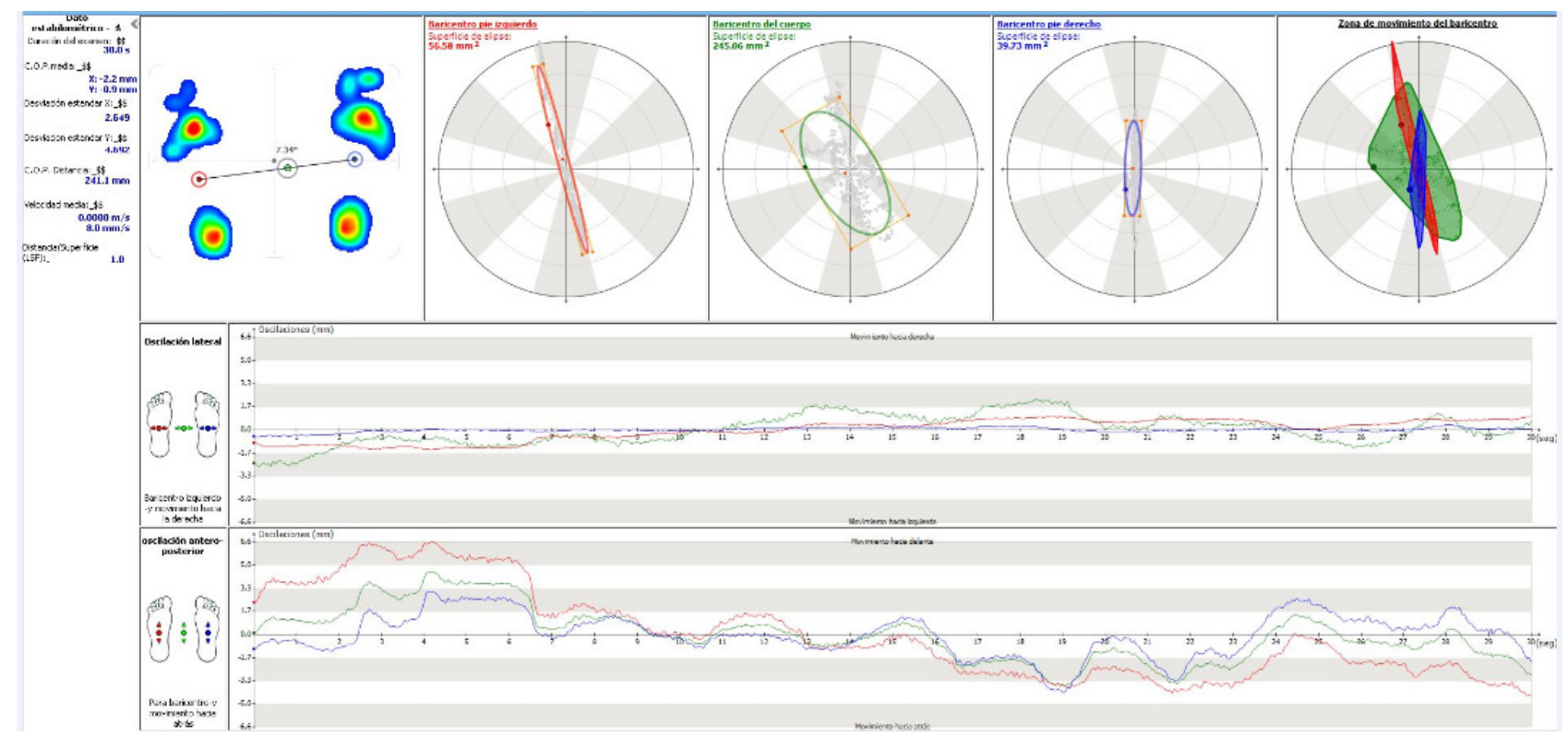

Fonte: Laboratorio Análisis de Movimiento - Universidad Autónoma de Manizales.

Figura 1 - Resultados estabilométricos pré-teste

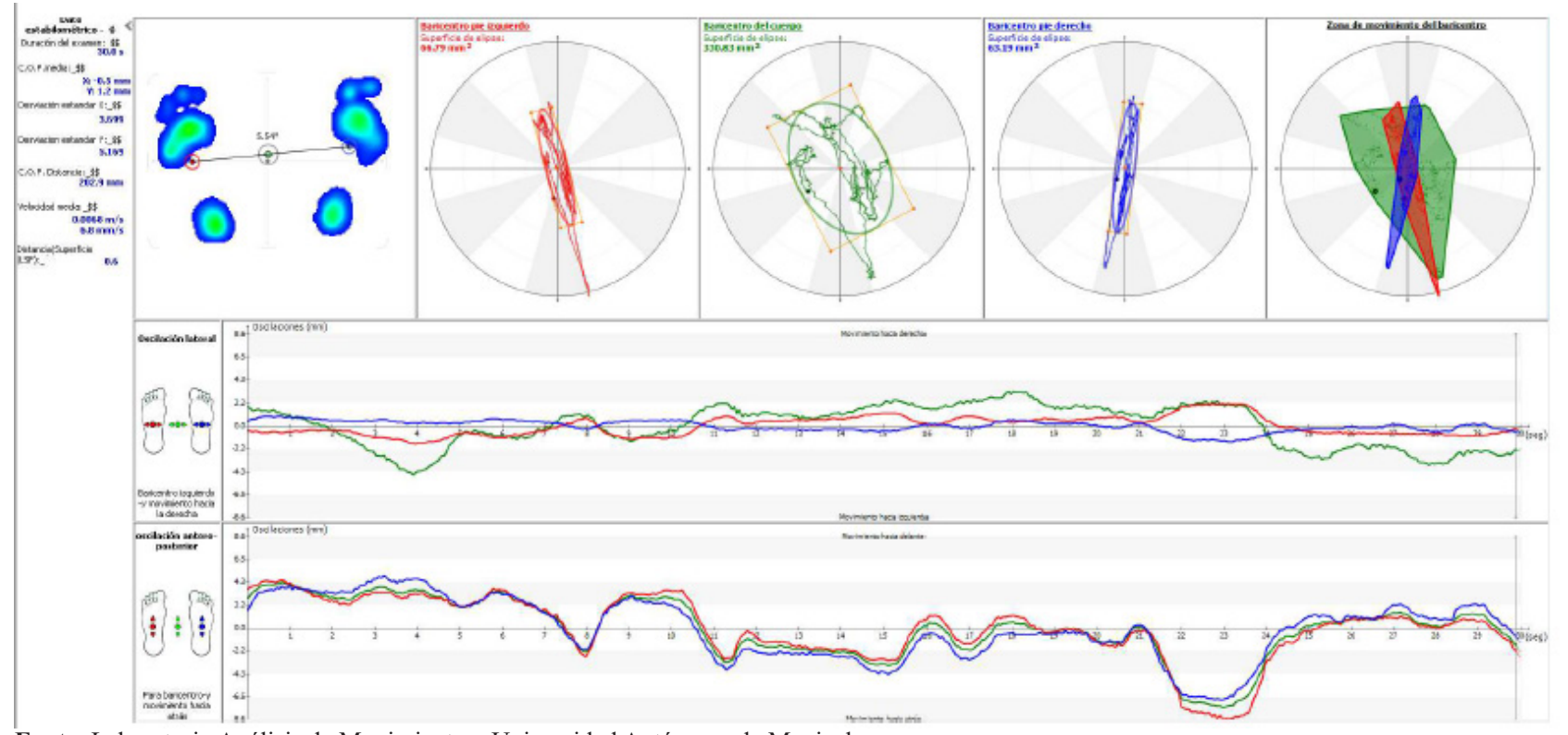

Fonte: Laboratorio Análisis de Movimiento - Universidad Autónoma de Manizales.

Figura 2 - Resultados estabilométricos pós-teste 


\section{DISCUSSÃO}

Após a aplicação do protocolo durante o tratamento fisioterapêutico, a paciente apresentou melhora em alguns aspectos da marcha. O equilíbrio também apresentou progresso sobre superfície estável com olhos fechados, como também em superfície instável com olhos abertos. Uma análise de como a AM pode ser otimizada em adultos pode trazer implicações teóricas e práticas importantes. A baropodometria pode contribuir no diagnóstico funcional dado por fisioterapeutas e a estabilometria possui capacidade de medir quantitativamente as variáveis associadas ao equilíbrio podendo oferecer validez às provas clínicas. Nesta pesquisa a paciente apresentou oscilações no equilíbrio pós-treino menores comparadas às pré-treino. Estes resultados corroboram com outros pesquisadores que investigaram indivíduos com a doença de Charcot-MarieTooth e mostraram, através da estabilometria, diferença na força muscular em todos os movimentos de tornozelos, o que leva a uma maior instabilidade de equilíbrio ${ }^{14}$. Um outro estudo realizado com paciente neurológico coletou dados de oscilações e pressão, pré-treino e pós-treino, em ortostatismo, com baropodometria e estabilometria e os resultados apontaram uma melhora no deslocamento látero-medial e ântero-posterior, aumentando o controle do tronco e diminuindo a chance de quedas ${ }^{15}$.

A marcha promove a independência durante AVD's e sua análise é importante para compreender as variáveis presentes na locomoção fundamentando o tratamento de doenças que comprometem o aparelho locomotor. Durante a deambulação, déficit em equilíbrio e propriocepção são apontados como importantes causadores de quedas em pacientes neurológicos, logo, há a necessidade de desenvolver intervenções fisioterapêuticas que melhorem efetivamente estes problemas ${ }^{16}$. O pós-teste mostrou avanços nos aspectos avaliados. Pouco se sabe sobre o efeito da prática em sujeitos com ELA e nada foi encontrado com ELP, no entanto, pesquisas têm sido desenvolvidas sobre o treinamento de marcha e equilíbrio direcionados a outras doenças desmielinizantes, que podem entrar em concordância com nossos achados.

Um estudo de caso investigou os efeitos da fisioterapia na marcha, no equilíbrio, na flexibilidade e no tônus muscular em paciente com esclerose múltipla (EM) e obteve resultados positivos após o tratamento ${ }^{17}$.

Fonte de financiamento: Não teve.

Conflito de interesses: Nada a declarar.
Uma pesquisa avaliou alterações motoras em paciente pós Síndrome de Guillain-Barré e constatou que as técnicas fisioterapêuticas utilizadas foram eficazes no processo de reabilitação e levaram a melhoras na força muscular, amplitude de movimentos, na marcha e no equilíbrio ${ }^{18}$. Almeida et al. ${ }^{19}$ avaliaram o equilíbrio de nove indivíduos com doença de Parkinson antes e após um programa fisioterapêutico e sugerem melhora entre os sujeitos avaliados. Na pesquisa de Marcon et al. ${ }^{20}$ foram estudadas as modificações no equilíbrio através da realidade virtual, pré e pós intervenção, e sua influência na qualidade de vida $(\mathrm{QV})$ em cinco indivíduos com $\mathrm{EM}$ e os resultados mostraram que o treino leva ao restabelecimento do equilíbrio e possibilita influenciar na QV na EM. Dois pacientes com Distrofia Muscular de Steinert foram separados, sendo um controle apenas com fisioterapia convencional e outro com fisioterapia e prática do Wii reabilitação, avaliados através da baropodometria e os resultados mostraram que a prática levou à melhora do equilíbrio ${ }^{21}$.

Observa-se na literatura que a reabilitação física baseada em prática e repetição (necessárias para que haja AM) em pacientes neurológicos, geralmente oferece boa efetividade ao tratamento, possibilitando que a utilização deste protocolo seja promissora em ELP.

\section{CONCLUSÃO}

A criação e aplicação do protocolo de intervenção baseado na AM levaram a avanços funcionais em marcha e equilíbrio de jovem com ELP. Esta é uma patologia rara e progressiva e a abordagem terapêutica deve ter início imediato para um melhor prognóstico. Apesar de ter sido desenvolvido de acordo com o quadro funcional de uma paciente específica, o protocolo poderá ser útil como uma base de consulta para a elaboração de outras propostas com objetivos semelhantes.

\section{LIMITAÇÕES DO ESTUDO}

Apesar dos resultados positivos, um estudo de caso não permite generalizar recomendações. Ensaios clínicos envolvendo amostras maiores poderiam demonstrar os benefícios deste protocolo e indicar bons níveis de significância estatística.

Agradecimentos: Ao Laboratório de Análise do Movimento, à Faculdade de Saúde e ao Programa de Fisioterapia da Universidad Autónoma de Manizales.

Participação dos autores: Silva Filho JA; Bernal MYP; Ruiz JC: Coleta de dados pré-teste. Silva Filho JA: Elaboração do protocolo de intervenção. García CIG: Aplicação do protocolo de intervenção. Bernal MYP; Ruiz JC: Coleta de dados pós-teste. Silva Filho JA: Pesquisa e análise bibliográfica. Silva Filho JA: Leitura e escrita do conteúdo. Bernal MYP; Ruiz JC: Orientação organizacional. Bernal MYP; Ruiz JC: Revisão do texto quanto à integridade e veracidade das fontes utilizadas. Silva Filho JA: Redação final e submissão do artigo. 


\section{REFERÊNCIAS}

1. Agosta F, Canu E, Inuggi A, Chiò A, Riva N, Silani V, Calvo A, Messina S, Falini A, Comi G, Filippi M. Resting state functional connectivity alterations in primary lateral sclerosis. Neurobiol Aging. 2013;1-10. doi: https://doi. org/10.1016/j.neurobiolaging.2013.09.041.

2. Flynn L, Stephen M, Floeter MK. Disease spread through contiguity and axonal tracts in primary lateral sclerosis. Muscle Nerve. 2014;49(3):439-441. doi: https://doi. org/10.1002/mus.24116.

3. Schweitzer AD, Liu T, Gupta A, Zheng K, Seedial S, Shtilbans A, Shahbazi M, Lange D, Wang Y, Tsiouris AJ. Quantitative susceptibility mapping of the motor cortex in amyotrophic lateral sclerosis and primary lateral sclerosis. AJR Am J Roentgenol. 2015;204(5):1086-92. doi: https:// doi.org/10.2214/AJR.14.13459.

4. Budrewicz S, Szewczyk P, Slotwinski K, Koszewicz M. Symptoms of degeneration of the pyramidal tracts in conventional magnetic resonance imaging and diffusion tensor imaging in a young woman with primary lateral sclerosis. J Postgrad Med. 2015;61(3):206-8. doi: https:// doi.org/10.4103/0022-3859.150901.

5. Stantland JM, Barohn RJ, Dimanchkie MM, Floeter MK, Misumoto H. Primary lateral sclerosis. Neurol Clin. 2015;33(4):749-60. doi: https://doi.org/10.1016/j. ncl.2015.07.007.

6. Ferreira TB, Silva NPO, Martins LJNS, Brito MAM, Cavalcanti FAC. Fisioterapia motora na esclerose lateral amiotrófica: estudo descritivo de quatro protocolos de intervenção. Rev Neurocienc. 2015;23(4):609-16. doi: https://doi.org/10.4181/RNC.2015.23.04.1076.08p.

7. Florindo M, Pedro R. O processo de aprendizagem motora e a neuroplasticidade. Salutis Scientia. 2014;6:19-26. Disponível em: https://www.academia.edu/23315525/O_processo_de_ aprendizagem_motora_e_a_neuroplasticidade.

8. Guevara CR, Lugo LH. Validez y confiabilidad de la escala de Tinetti para población colombiana. Rev Colombiana Reumatol. 2012;19(4):218-33. doi: https://doi.org/10.1016/ S0121-8123(12)70017-8.

9. Fitzgerald B. A review of the sharpened Romberg test in diving medicine. SPUMS J. 1996;26(3):142-6.

10. Barbalaco L, Abudarham J, Argento F, Cazurro E, Dilascio S, Di Prinzio F, et al. Validación del Timed Up and Go test como predictor de riesgo de caídas en sujetos con artritis reumatoidea. Parte II: validez concurrente y predictive. Rev Argentina Reumatol. 2019;30(4):3-9. Disponível em: http:// www.revistasar.org.ar/revistas/2019/n4/2_articulo\%20 original.pdf.

11. Alfonso-Mora ML. Metric properties of the "timed get up and go - modiefied version" test, in risk assessment of falls in active women. Colombia Méd (Cali). 2017;48(1):19-24. Available from: https:/www.ncbi.nlm.nih.gov/pmc/articles/ PMC5438224/.
12. Curcio CL, Gómez JF, Galeano IC. Validezy reproducibilidad de medidas de evaluación funcional basadas en la ejecución. Rev Esp Geriatr Gerontol. 2000;35(2):82-8. Disponível em: https://www.elsevier.es/es-revista-revistaespanola-geriatria-gerontologia-124-articulo-validezreproducibilidad-medidas-evaluacion-funcional-13011691.

13. Duncan PW, Weiner DK, Chandler J, Studenski S. Functional reach: a new clinical measure of balance. J Gerontol. 1990;45(6):192-7. doi: https://doi.org/10.1093/ geronj/45.6.m192.

14. Costa IMPF, Araújo AAS. Avaliação do equilíbrio, da força muscular e da funcionalidade de indivíduos com a doença de Charcot-Marie-Tooth [Tese]. Aracaju: Universidade Federal de Sergipe; 2016. Disponível em: http://bdtd.ibict.br/vufind/ Record/UFS-2_7b3da0f64cbe25ceb788e03551a08ae0.

15. Mello GCV, Silva GC, Fortes JPA, Nascimento Filho PC, Brasileiro IC, Santos Júnior FFU. Equilíbrio estático por baropodometria em paciente com ataxia cerebelar após tratamento com neurofeedback. Fisioter Bras. 2017;18(2):249-56. Disponível em: https:// portalatlanticaeditora.com.br/index.php/fisioterapiabrasil/ article/view/802/2081.

16. Lessa HT. Aprendizagem motora e doença de Parkinson: revisão de fatores influentes no equilíbrio e na propriocepção. Rev Neuroci. 2013;21(2):308-12. https://doi.org/10.34024/ rnc.2013.v21.8187.

17. Garcia T, Mederdrut EM, Fontes SV. Efeitos da fisioterapia com trampolim na esclerose múltipla: um estudo de caso. Rev Bras Ciên Saúde. 2008;17(3):18-25. doi: http://dx.doi. org/10.13037/rbcs.vol6n17.355.

18. Sá BP, Grave MTQ, Périco E, Bohrer TRJ. Avaliação e tratamento de sequelas motoras pós síndrome de Guillain-Barré (SGB): estudo de caso. Cad Pedagógico. 2015;12(3):131-9. Disponível em: http://www.univates.br/ revistas/index.php/cadped/article/view/974/962.

19. Almeida IA, Bueno MEB, Andrello ACR, Batistetti CL, Lemes LB, Barboza NM, Melo LB, Santos SMS. Fisioterapia baseada no treinamento de dupla tarefa no equilíbrio de indivíduos com doença de Parkinson. Saúde (Santa Maria). 2015;41(2):71-80. doi: http://dx.doi. org/10.5902/2236583413885.

20. Marcon CLV, Soares JC, Oliveira GC, Mota CB, Trevisan CM. Efeitos da realidade virtual sobre o equilíbrio e a qualidade de vida em pacientes portadores de esclerose múltipla. Rev Bras Biomecanica. 2015;16(34).

21. Jeremias GC, Ferraz C, Vicente E. Avaliação baropodométrica e do equilíbrio em pacientes com Distrofia Muscular de Steinert antes e após a prática do WII reabilitação. Rev Inova Saúde. 2017;6(2):114-31. doi: http://dx.doi.org/10.18616/is.v6i2.2428.

Recebido: 03.06.2019

Aceito: 21.01.2021 